\title{
FIB-4 index associated with mortality risk of patients with systemic lupus erythematosus: a large retrospective cohort study
}

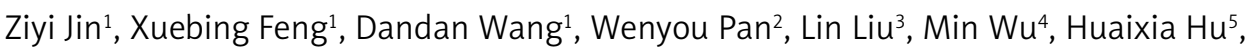 \\ Xiang Ding ${ }^{6}$, Hua Wei ${ }^{7}$, Yaohong Zou ${ }^{8}$, Xian Qian', Meimei Wang ${ }^{10}$, Jian $\mathrm{Wu}^{11}$, Juan Tao ${ }^{12}$, Jun $\operatorname{Tan}^{13}$, \\ Zhanyun $\mathrm{Da}^{14}$, Miaojia Zhang ${ }^{15}$, Jing $\mathrm{Li}^{16}$, Lingyun Sun ${ }^{1}$
}

\author{
${ }^{1}$ Department of Rheumatology and Immunology, the Affiliated Drum Tower Hospital \\ of Nanjing University Medical School, Nanjing, China \\ 2Department of Rheumatology, Huai'an First People's Hospital, Huai'an, China \\ ${ }^{3}$ Department of Rheumatology, Xuzhou Central Hospital, Xuzhou, China \\ ${ }^{4}$ Department of Rheumatology, the Third Affiliated Hospital of Soochow University, \\ Changzhou, China \\ Department of Rheumatology, Lianyungang Second People’s Hospital, Lianyungang, \\ China \\ ${ }^{6}$ Department of Rheumatology, Lianyungang First People's Hospital, Lianyungang, \\ China \\ 'Department of Rheumatology, Northern Jiangsu People's Hospital, Yangzhou, China \\ ${ }^{8}$ Department of Rheumatology, Wuxi People's Hospital, Wuxi, China \\ ${ }^{9}$ Department of Rheumatology, Jiangsu Province Hospital of TCM, Nanjing, China \\ ${ }^{10}$ Department of Rheumatology, Southeast University Zhongda Hospital, Nanjing, China \\ ${ }^{11}$ Department of Rheumatology, the First Affiliated Hospital of Soochow University, \\ Suzhou, China \\ ${ }^{12}$ Department of Rheumatology, Wuxi TCM Hospital, Wuxi, China \\ ${ }^{13}$ Department of Rheumatology, Zhenjiang First People's Hospital, Zhenjiang, China \\ ${ }^{14}$ Department of Rheumatology, Affiliated Hospital of Nantong University, Nantong, \\ China \\ ${ }^{15}$ Department of Rheumatology, Jiangsu Province Hospital, Nanjing, China \\ ${ }^{16}$ Department of Rheumatology, Affiliated Hospital of Jiangsu University, Jiangsu, \\ China
}

Submitted: 20.01 .2020

Accepted: 19.05 .2020

Arch Med Sci

DOI: https://doi.org/10.5114/aoms.2020.101049

Copyright $\odot 2020$ Termedia \& Banach

\section{Abstract}

Introduction: A considerable proportion of patients with systemic lupus erythematosus (SLE) have liver enzyme abnormalities. We evaluated whether fibrosis-4 (FIB-4) was associated with increased mortality of SLE patients.

Material and methods: A multicenter retrospective cohort study was conducted based on medical records of first-admission SLE patients who were hospitalized during 1999-2009 in Jiangsu province. FIB-4 was estimated by age, platelet (PLT) count, alanine aminotransferase (ALT), aspartate aminotransferase (AST), and FIB-4. A Cox model and a restricted cubic spline (RCS) model were used to estimate hazard ratio (HR) and $95 \%$ confidence interval $(95 \% \mathrm{Cl})$

Results: During up to 15 years of follow-up, 226 deaths were observed among 2331 SLE patients. Increased overall mortality was associated with abnormal groups of PLT, ALT, AST, and high FIB-4 ( $\geq 1.92)$, with adjusted HRs $(95 \% \mathrm{Cl})$ of 1.58 (1.19-2.11), 1.54 (1.14-2.07), 1.58 (1.17-2.12), and 1.88 (1.42-2.50), respectively. Moreover, significant dose-response relationships were found between mortality of SLE and those indexes, and it was nonlinear for PLT $(p<0.001)$, ALT $(p=0.046)$ and FIB-4 $(p<0.001)$, but not AST $(p=0.109)$. In cause-specific analyses, high FIB-4 index was directly associated

\author{
Corresponding author: \\ Lingyun Sun MD \\ Department of Rheumatology \\ and Immunology \\ The Affiliated Drum Tower \\ Hospital \\ Nanjing University Medical \\ School \\ 321 Zhongshan Road \\ Nanjing 210008, China \\ Phone: +86-25-6818-2422 \\ Fax: +86-25-6818-2428 \\ E-mail: lingyunsun@nju. \\ edu.cn
}


with death from liver failure and indirectly associated with other death from infection and neuropsychiatric impairment. We found no significant differences of areas under curves (AUCs) between FIB-4 index and the disease activity index score of SLE.

Conclusions: This is the first report to describe the nonlinear association between FIB-4 index and increased mortality of SLE patients. The FIB-4 index may be used as an independent prognostic indicator for SLE patients.

Key words: liver enzymes, FIB-4, hazard ratio, mortality, systemic lupus erythematosus.

\section{Introduction}

Systemic lupus erythematosus (SLE) is a chronic multisystem autoimmune disease and affects several organs, including the liver [1]. It is clinically frequent with abnormal liver function tests, and the prevalence of developing liver enzyme abnormalities is about $25-60 \%$ among patients with SLE [2-4]. The causes of liver enzyme abnormalities are numerous, such as drug toxicity, viral infections, alcohol abuse, and fatty infiltration, but either disease activity or drugs can be the main etiology among SLE patients [4-6]. With improvement of therapy, ten-year survival rates of patients with SLE have been more than $80 \%$; moreover, patients with chronic liver injury are more prone to undergo the process from fibrosis to cirrhosis, liver failure, and even hepatocellular carcinoma (HCC) [7-10]. Moreover, malignancies, especially liver cancer, have become the leading causes of death among Western patients with SLE [11-13]. Thus, understanding the role of liver function in prognosis of SLE patients is important in developing an adaptive treatment strategy.

Few SLE patients have liver biopsy and imagological examination for liver function examination in routine clinical practice, meaning they are not suitable for screening of clinically significant liver function for patients with SLE [14]. Fortunately, several scoring systems methods based on simple clinical parameters and blood tests for assessing liver fibrosis have been developed, whereas the Fibrosis-4 (FIB-4) index is the better predictor $[15,16]$. The FIB-4 index is developed from age, alanine aminotransferase (ALT) and aspartate aminotransferase (AST) levels, and the platelet (PLT) count, which has been applied in predicting overall and specific mortality of patients in several cohort studies [17-20]. Moreover, the FIB-4 index is much simpler than the disease activity index (SLEDAI) score, which is currently used by rheumatologists to evaluate the outcomes of patients with SLE [21]. However, so far no studies have explored the association between the FIB-4 index and mortality of patients with SLE. Therefore, we analyzed data collected from the Jiangsu SLE Cohort (JSC) Study, a large multicenter retrospective cohort study in China $[22,23]$. Our study aimed to explore the potential prognostic role of the FIB-4 index for patients with SLE. In addition, we compared it with the SLEDAI score for prognosis in SLE.

\section{Material and methods}

\section{Study design and participants}

The detailed study design of the JSC Study has been described previously [22, 23]. To summarize, the JSC Study is a large-scale, multicenter retrospective cohort study in China. Since 2010, under the organization of the Jiangsu Rheumatology Association, the Lupus Collaborative Group has built up the cohort of patients with SLE from hospitals in Jiangsu province, China.

All patients were hospitalized during 19992009 in Jiangsu province and those with missing or incomplete medical records were excluded. Eligible patients were those who met at least 4 classification criteria of SLE revised and updated by the American College of Rheumatology [24, 25]. A follow-up for survival status of patients with SLE was conducted among those who had medical records for the first time/admission. Up to 2015, we reviewed and enrolled 2451 valid medical records and twice carried out the follow-up, in 2010 and 2015. In total, we observed 226 deaths, comprising 166 deaths in 2010 and 60 deaths in 2015. We further excluded 120 patients because their FIB-4 index cannot be calculated due to AST, ALT and/or PLT being missing.

This study was performed in 2015 and was exempt from ethics approval requirements, since ethical review of research involving medical records or biospecimens was firstly required in 2016 according to national legislation from the National Health and Family Planning Commission of China [26].

\section{Data collection and definition}

We built a website (http://sys.91sqs.net/sle/Index/index.html) to collect and manage data from medical records of patients with SLE from different hospitals. We also extracted data from all medical records of first admission, including demographic characteristics (gender, age), diagnosed information, disease activity and damage, organ involvements, laboratory tests, and treatments. 
The SLEDAI score was calculated to assess disease activity and was categorized into four grades, including almost no activity (SLEDAI $=0-4$ ), mild activity (SLEDAI $=5-9$ ), moderate activity $($ SLEDAI $=10-14)$, and high activity (SLEDAI $\geq 15)$ [21]. The FIB-4 index was calculated according to the following formula: (age [years] $\times$ AST [IU/I])/ $\left(\mathrm{PLT}\left[\times 10^{9} / \mathrm{I}\right] \times \mathrm{ALT}[\mathrm{IU} / \mathrm{I}]^{1 / 2}\right)[27]$. We combined all diseases other than SLE as comorbidities. Accumulated organ damage in SLE was evaluated by the Systemic Lupus International Collaborating Clinics (SLICC)/American College of Rheumatology (ACR) Damage Index (SDI) [28]. The following values were considered normal: hemoglobin $\geq 110 \mathrm{~g} / \mathrm{l}$ for female or $\geq 120 \mathrm{~g} / \mathrm{l}$ for male; white blood cells $4-10 \times 10^{9} / \mathrm{l}$, PLT 100-300 × 10\% /, ALT < $40 \mathrm{lU} / \mathrm{l}$, AST $<40 \mathrm{IU} / \mathrm{l}$, serum albumin $\geq 35 \mathrm{~g} / \mathrm{l}, 24 \mathrm{~h}$-urinary proteins $<500 \mathrm{mg}$, blood urea nitrogen $2.86-$ $7.14 \mathrm{mmol} / \mathrm{l}$, serum creatinine $\leq 133 \mu \mathrm{mol} / \mathrm{l}$, antidsDNA negative, antinuclear antibody negative, anti-Sm negative, C3 $\geq 0.8 \mathrm{~g} / \mathrm{l}, \mathrm{C} 4 \geq 0.2 \mathrm{~g} / \mathrm{l}$.

\section{Statistical analysis}

Statistical analyses were performed using Statistical Analysis Software (SAS) version 9.3 (SAS Institute, Inc., Cary, North Carolina, USA) and the program R (Version 3.5.3, R core team). Continuous variables were expressed as the median (quartile) and compared using the Mann-Whitney $U$ test, or as mean and standard deviation (SD) compared using the $t$ test. Categorical variables were expressed as the number (percentage), and descriptive analyses on the basis of the $\chi^{2}$ test were carried out for the distribution differences between deaths and censored survivals.

Kaplan-Meier survival analysis and log-rank tests were used for the initial survival analysis. Time in years was calculated from the diagnosis date of the patient to the date of death for deaths and to the date of the last follow-up for censored survivors. For survival analysis, two approaches were employed to explore the influence of the FIB-4 index on the risk of mortality of SLE. Firstly, we used Cox proportional-hazards regression models regression to estimate crude and adjusted hazard ratio $(\mathrm{HR})$ and $95 \%$ confidence interval $(95 \% \mathrm{Cl})$. The FIB-4 index was classified into two categories according to the optimal Youden index-based cutoff point value by receiver operating characteristic (ROC) curve. The cut-off value for the FIB-4 index was 1.92, which was within the two cut-off values for three-way classification of the FIB-4 index among patients with human immunodeficiency virus/hepatitis C virus (HIV/HCV) coinfection (1.45 and 3.25) and nonalcoholic fatty liver disease (NAFLD) (1.30 and 2.67) [16, 27]. In cause-specific mortality analyses, subjects who did not die from the cause of interest were censored at the date of death. Secondly, an restricted cubic spline (RCS) model was used to estimate the adjusted dose-response relationship between FIB-4 index and mortality of SLE patients. Furthermore, we performed ROC curve analysis for estimating and comparing time-dependent areas under curves (AUCS) between FIB-4 index and SLEDAI score [29].

The following factors were included in the models as potential confounders: gender (male - 1, female -0), SLEDAI score at admission (continuous), comorbidities (yes -1 , no - 0), glucocorticoid treatment on admission (yes - 1 , no - 0), immunosuppressive treatment on admission (yes -1 , no - 0). We also performed stratified analysis across clinical factors of patients with SLE patients.

\section{Results}

\section{Characteristics of study subjects}

Table I shows demographic and clinical characteristics of patients with SLE among deaths and censored survivals. Of all 2331 participants, 92.5\% (2156) were female and the median (quartiles) age was 32.02 (23.61, 40.61). The median (quartiles) SLEDAI score at admission was 16.0 $(11.0,22.0)$ for deaths and $13.0(9.0,19.0)$ for censored survivals $(p<0.001)$. Deaths and censored survivals also differed in serum hemoglobin, PLT, AST, FIB-4 index, serum albumin, and $24 \mathrm{~h}$ urinary proteins, except age, body mass index (BMI) and white blood cells. For the distribution, no statistically significant differences were observed between deaths and censored survivals in gender, overall comorbidities, glucocorticoid drugs used before admission and on admission, mucocutaneous and musculoskeletal organ involvement, or positive autoantibodies of anti-dsDNA and antinuclear antibody, except immunosuppressive drugs used before admission ( $p=0.001)$ and on admission $(p<0.001)$, neuropsychiatric $(p<0.001)$ and cardiopulmonary $(p<0.001)$ organ involvement, and anti-Sm positive autoantibodies $(p=0.006)$.

\section{Hazard ratios for mortality}

The hazard ratios of mortality of patients with SLE for SLEDAI score and liver function are shown in Table II. High SLEDAl score at admission was confirmed as a strong risk factor for mortality of SLE patients with adjusted $\mathrm{HR}$ and $\mathrm{Cl}$ of 2.55 (95\% $\mathrm{Cl}$ : 1.68-3.00) for a SLEDAl score of 15 or more. After adjusting for confounding factors, positive associations were found between mortality of SLE and hepatitis, and abnormal serum albumin, with adjusted HR of 2.27 (95\% Cl: 1.23-4.21) and 2.20 (95\% Cl: 1.57-3.09), respectively. Drugs used before admission was not associated with mortality of SLE (adjusted HR = 1.29, 95\% Cl: 0.96-1.74). Compared with the group with normal values, $a b$ - 
Z. Jin, X. Feng, D. Wang, W. Pan, L. Liu, M. Wu, H. Hu, X. Ding, H. Wei, Y. Zou, X. Qian, M. Wang, J. Wu, J. Tao, J. Tan, Z. Da, M. Zhang, J. Li, L. Sun

Table I. Characteristics of hospitalized patients with systemic lupus erythematosus (SLE) from Jiangsu cohort study

\begin{tabular}{|c|c|c|c|}
\hline Variables & Censored & Death & $P$-value \\
\hline Total & 2105 & 226 & \\
\hline \multicolumn{4}{|c|}{ Median (quartiles) or Mean \pm SD } \\
\hline Age (years) & $32.0(23.7,40.3)$ & $32.8(23.5,43.0)$ & 0.345 \\
\hline $\mathrm{BMI}\left(\mathrm{kg} / \mathrm{m}^{2}\right)$ & $21.5 \pm 3.2$ & $22.5 \pm 3.6$ & 0.217 \\
\hline SLEDAI score at admission & $13.0(9.0,19.0)$ & $16.0(11.0,22.0)$ & $<0.001$ \\
\hline \multicolumn{4}{|l|}{ Laboratory tests: } \\
\hline Serum hemoglobin & $104.0(89.0,118.0)$ & $94.0(74.0,109.0)$ & $<0.001$ \\
\hline White blood cells & $4.2(3.0,6.3)$ & $4.2(2.9,7.1)$ & 0.932 \\
\hline $\operatorname{PLT}\left(10^{9} / \mathrm{l}\right)$ & $139.0(92.0,190.0)$ & $109.0(64.0,154.0)$ & $<0.001$ \\
\hline $\operatorname{ALT}(I U / l)$ & $23.0(16.0,38.0)$ & $26.5(16.0,45.0)$ & 0.022 \\
\hline AST (IU/l) & $25.5(18.0,39.8)$ & $29.9(18.3,55.0)$ & 0.003 \\
\hline FIB-4 index & $1.3(0.7,2.5)$ & $2.0(0.9,4.7)$ & $<0.001$ \\
\hline Serum albumin (g/l) & $34.0(28.8,38.6)$ & $30.2(24.6,35.0)$ & $<0.001$ \\
\hline $24 \mathrm{~h}$ urinary proteins (g) & $4.9(3.7,6.9)$ & $6.1(4.2,11.2)$ & $<0.001$ \\
\hline \multicolumn{4}{|c|}{ Number and proportion \% } \\
\hline Gender (female) & $1953(92.8)$ & $203(89.8)$ & 0.109 \\
\hline \multicolumn{4}{|l|}{ Comorbidities: } \\
\hline All & $209(9.9)$ & $31(13.7)$ & 0.075 \\
\hline Diabetes & $67(3.2)$ & $11(4.9)$ & 0.181 \\
\hline Hypertension & $35(1.7)$ & $10(4.4)$ & 0.004 \\
\hline Secondary Sjögren's syndrome & $34(1.6)$ & $4(1.8)$ & 0.861 \\
\hline $\begin{array}{l}\text { Pulmonary or upper respiratory } \\
\text { infections }\end{array}$ & $34(1.6)$ & $2(0.9)$ & 0.398 \\
\hline Others & $57(2.7)$ & $8(3.5)$ & 0.470 \\
\hline \multicolumn{4}{|l|}{ Drugs used before admission: } \\
\hline Glucocorticoids & $5(0.2)$ & $2(0.9)$ & 0.091 \\
\hline Immunosuppressive & $521(24.8)$ & $78(34.5)$ & 0.001 \\
\hline \multicolumn{4}{|l|}{ Treatments on admission: } \\
\hline Glucocorticoids & $1966(93.4)$ & $213(94.2)$ & 0.622 \\
\hline Immunosuppressive & $1561(74.2)$ & $135(59.7)$ & $<0.001$ \\
\hline \multicolumn{4}{|l|}{ Organ involvement: } \\
\hline Mucocutaneous & $1384(65.7)$ & $143(63.3)$ & 0.457 \\
\hline Neuropsychiatric & $109(5.2)$ & $32(14.2)$ & $<0.001$ \\
\hline Musculoskeletal & $1167(55.4)$ & $111(49.1)$ & 0.069 \\
\hline Cardiopulmonary & $426(20.2)$ & $82(36.3)$ & $<0.001$ \\
\hline \multicolumn{4}{|l|}{ Positive autoantibodies: } \\
\hline Anti-dsDNA & $1142(63.1)$ & $134(67.3)$ & 0.242 \\
\hline Antinuclear antibody & $1865(93.3)$ & $189(93.1)$ & 0.896 \\
\hline Anti-Sm & $648(34.3)$ & $48(24.6)$ & 0.006 \\
\hline \multicolumn{4}{|l|}{ Abnormal complement: } \\
\hline $\mathrm{C} 3$ & $1373(73.3)$ & $143(73.7)$ & 0.903 \\
\hline $\mathrm{C} 4$ & $1015(64.2)$ & $108(66.3)$ & 0.609 \\
\hline
\end{tabular}

BMI - body mass index, SLEDAI - SLE Disease Activity Index, PLT - platelet, ALT - alanine aminotransferase, AST - aspartate aminotransferase, FIB-4 - fibrosis-4. 
Table II. Hazard ratios for survival of patients with systemic lupus erythematosus (SLE) according to SLEDAI score and liver function

\begin{tabular}{|c|c|c|c|c|}
\hline Variables & $\begin{array}{c}\text { All } \\
N=2331 \\
n(\%)\end{array}$ & $\begin{array}{c}\text { Death } \\
N=226 \\
n(\%)\end{array}$ & HR $(95 \% \mathrm{Cl})$ & $\begin{array}{c}\text { Adjusted } \\
\text { HR }(95 \% \mathrm{Cl})^{\mathrm{a}}\end{array}$ \\
\hline \multicolumn{5}{|c|}{ SLEDAI score at admission: } \\
\hline $0-14$ & $1273(54.6)$ & $90(39.8)$ & 1.00 & 1.00 \\
\hline$\geq 15$ & $1058(45.4)$ & $136(60.2)$ & $2.15(1.61-2.86)$ & $2.25(1.68-3.00)$ \\
\hline \multicolumn{5}{|l|}{ Hepatitis: } \\
\hline No & $2273(97.5)$ & $215(95.1)$ & 1.00 & 1.00 \\
\hline Yes & $58(2.5)$ & $11(4.9)$ & $2.48(1.35-4.55)$ & $2.27(1.23-4.21)$ \\
\hline \multicolumn{5}{|c|}{ Drugs used before admission: } \\
\hline No & $1728(74.1)$ & $147(65.0)$ & 1.00 & 1.00 \\
\hline Yes & $603(25.9)$ & $79(35.0)$ & $1.26(0.94-1.69)$ & $1.29(0.96-1.74)$ \\
\hline \multicolumn{5}{|c|}{ Serum albumin $(\mathrm{g} / \mathrm{l})$ : } \\
\hline$\geq 35$ & $993(42.6)$ & $57(25.2)$ & 1.00 & 1.00 \\
\hline$<35$ & $1294(55.5)$ & $169(74.8)$ & $2.60(1.87-3.61)$ & $2.20(1.57-3.09)$ \\
\hline \multicolumn{5}{|l|}{$\operatorname{PLT}\left(10^{9} / \mathrm{l}\right):$} \\
\hline $100-300$ & $1612(69.2)$ & $125(55.3)$ & 1.00 & 1.00 \\
\hline$<100$ or $>300$ & $719(30.8)$ & $101(44.7)$ & $1.84(1.39-2.43)$ & $1.58(1.19-2.11)$ \\
\hline \multicolumn{5}{|l|}{$\operatorname{ALT}(\mathrm{IU} / \mathrm{l}):$} \\
\hline$<40$ & $1757(75.4)$ & $151(66.8)$ & 1.00 & 1.00 \\
\hline$\geq 40$ & $574(24.6)$ & $75(33.2)$ & $1.54(1.14-2.07)$ & $1.54(1.14-2.07)$ \\
\hline \multicolumn{5}{|l|}{ AST (IU/I): } \\
\hline$<40$ & $1729(74.2)$ & $148(65.5)$ & 1.00 & 1.00 \\
\hline$\geq 40$ & $602(25.8)$ & $78(34.5)$ & $1.69(1.26-2.26)$ & $1.58(1.17-2.12)$ \\
\hline \multicolumn{5}{|l|}{ FIB-4 index: } \\
\hline$<1.92$ & $1531(65.7)$ & $109(48.2)$ & 1.00 & 1.00 \\
\hline$\geq 1.92$ & $800(34.3)$ & $117(51.8)$ & $2.11(1.60-2.79)$ & $1.88(1.42-2.50)$ \\
\hline
\end{tabular}

${ }^{a}$ Adjusted for sex (male - 1, female - 0), age (continuous, except for variables of FIB-4 index), SLEDAI score at admission (continuous, except for variables of SLEDAI score at admission), comorbidities (yes - 1, no - 0), glucocorticoid treatment on admission (yes - 1, no -0), immunosuppressive treatment on admission (yes - 1, no - 0). SLEDAI - SLE Disease Activity Index, PLT - platelet, ALT - alanine aminotransferase, AST - aspartate aminotransferase, FIB-4 - fibrosis-4.

normal groups of PLT, ALT and AST, and high FIB-4 $(\geq 1.92)$ were positively associated with mortality of SLE, with adjusted HR of 1.58 (95\% Cl: 1.192.11), 1.54 (95\% Cl: 1.14-2.07), $1.58(95 \% \mathrm{Cl}$ : $1.17-2.12)$, and 1.88 (95\% Cl: $1.42-2.12)$, respectively. Better survival was observed for those who had low FIB-4, compare with those who had high FIB-4, and log-rank $p$ value was less than 0.001 (Figure 1). After adjusting for potential factors, a positive association between FIB-4 index and mortality of patients with SLE was observed consistently across all strata, except for male, normal hemoglobin, abnormal PLT, normal serum albumin, and antinuclear antibody negative (Figure 2).

\section{Dose-response relationships}

In the RCS model, significant dose-response relationships ( $p$ values for overall association) were found between increased mortality of SLE patients and elevated level of PLT $(p<0.001)$, ALT $(p=$ $0.043)$, AST $(p<0.001)$ and FIB-4 index $(p<0.001)$. Also it was nonlinear ( $p$ values for nonlinear association) for ALT ( $p=0.046)$, PLT $(p<0.001)$ and FIB-4 $(p<0.001)$, but not AST $(p=0.109)$. (Figure 3 ).

\section{Factors associated with high FIB-4 index}

The risk factors associated with high FIB-4 index were gender, age, SLEDAI score at admission, 


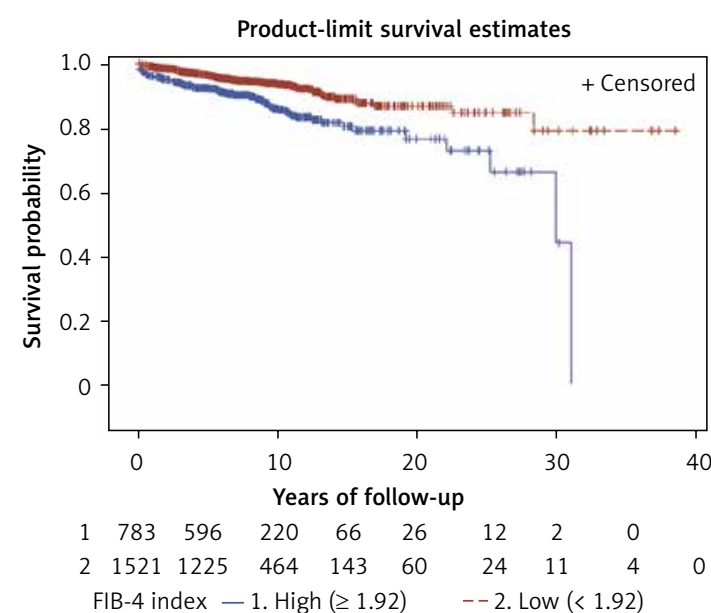

Figure 1. Kaplan-Meier analysis and log-rank $P$-values $(<0.001)$ for FIB-4 index in patients with systemic lupus erythematosus (SLE)

$24 \mathrm{~h}$ urinary proteins, neuropsychiatric and cardiopulmonary organ involvement, and abnormal complement of C3 and C4 (Table III).

\section{Cause-specific mortality}

Among 226 deaths in this cohort, the main causes of death were infection $(n=64,28.3 \%)$, cardiopulmonary involvement ( $n=38,16.8 \%)$, neuropsychiatric impairment ( $n=37,16.4 \%)$, and renal failure $(n=33,14.6 \%)$. A group of other causes of death $(n=54,23.9 \%)$ includes hematologic involvement, macrophage activation syndrome, tumor, suicide and unknown (recorded as cardiopulmonary failure or multiple organ failure).

In particular, 7 patients died of gastrointestinal complications, including liver failure $(n=4)$, gastrointestinal bleeding $(n=2)$, and pancreatitis $(n=1)$.

Figure 4 shows the association between FIB-4 index and mortality from each specific cause among patients with SLE. High FIB-4 index ( $\geq 1.92)$ was significantly associated with increased mortality from infection and neuropsychiatric impairment, with adjusted HR of 2.14 (95\% Cl: 1.27-3.61) and 2.12 (95\% Cl: 1.08-4.18), respectively. Elevated mortality from renal failure (adjusted $\mathrm{HR}=1.08$, 95\% Cl: 0.51-2.27) and cardiopulmonary involvement (adjusted $\mathrm{HR}=1.66,95 \% \mathrm{Cl}$ : 0.85-3.24) were observed in subjects with a high FIB-4 index compared to those with a low FIB-4 index, but it was not statistically significant. For subjects with a high FIB-4 index, adjusted HR of 2.05 (95\% Cl: 1.03-4.06) for risk of mortality from other causes increased to 2.57 (95\% Cl: 1.33-4.94) when specific mortality from liver diseases was included in the analysis.

\section{Comparison of performance of FIB-4 index and SLEDAI score}

The joint effects of FIB-4 index and SLEDAI score at admission on hazard ratios for surviv- al of patients with SLE are described in Table IV. No significant difference was observed between SLEDAI score and PLT ( $p=0.339)$, ALT $(p=0.846)$, AST $(p=0.785)$ or FIB-4 index $(p=0.996)$ for the association with mortality of SLE patients. Moreover, Figure 5 demonstrates ROC curve analysis for estimating and comparing time-dependent AUCS between FIB-4 index and SLEDAI score at (A) 1, (B) 5, (C) 10 and (D) 15 years. We found no significant difference of AUCs between FIB-4 index and SLEDAI score, with $p$ values for the comparison of AUCs at 1, 5, 10 and 15 years between FIB-4 index and SLEDAI score of 0.263, 0.654, 0.250, and 0.074 , respectively.

\section{Discussion}

In this large-scale multicenter retrospective cohort study, we found that higher FIB-4 index was positively associated with increased overall mortality of SLE with a nonlinear dose-response relationship. Moreover, ROC curve analysis indicated no significant differences of AUCs between FIB-4 index and SLEDAI score.

Up to now, there are no studies focusing on the association between the FIB-4 index and mortality of patients with SLE. This can be easily understood as few prospective studies have enough statistical power to predict mortality of liver-related diseases by using liver function indexes [17, 30, 31]. Consistent with our results that high FIB-4 index $(\geq 1.92$ vs. $<1.92$ ) was associated with increased overall mortality of SLE patients with adjusted $\mathrm{HR}$ of 1.88 (95\% Cl: 1.42-2.50), accumulated evidence has shown that the FIB-4 index may be used as a prognostic marker [17-20, 30, 31]. Recently, in the United States, in a population-based prospective study, with up to 23 years of follow-up of 14,841 viral hepatitis-negative adult participants, researchers observed that a high FIB-4 index of more than 2.67 was associated with increased overall mortality with age adjusted HR of 1.38 (95\% Cl: 1.21-1.58) [18]. Moreover, for the cause-specific mortality risk, mortality from cardiovascular disease was only higher with a high FIB-4 index ( $\mathrm{HR}=1.32$, 95\% Cl: 1.05-1.66), while mortality from liver disease was higher with an intermediate $(\mathrm{HR}=3.49,95 \% \mathrm{Cl}: 1.52-8.03)$ or high $(\mathrm{HR}=42.45,95 \% \mathrm{Cl}: 14.93-120.72) \mathrm{FIB}-4$ index in multivariate-adjusted analyses, but mortality from neoplasms and diabetes were elevated with neither intermediate nor high FIB-4 indexes [18]. In a previous study using the same cohort with 18 years of follow-up, FIB-4 index was also found to be independently associated with increased overall mortality, primarily from cardiovascular disease but not from liver disease [19]. In another recent cohort study of 2075 patients with chronic hepatitis B virus (HBV) infection, a high FIB-4 in- 


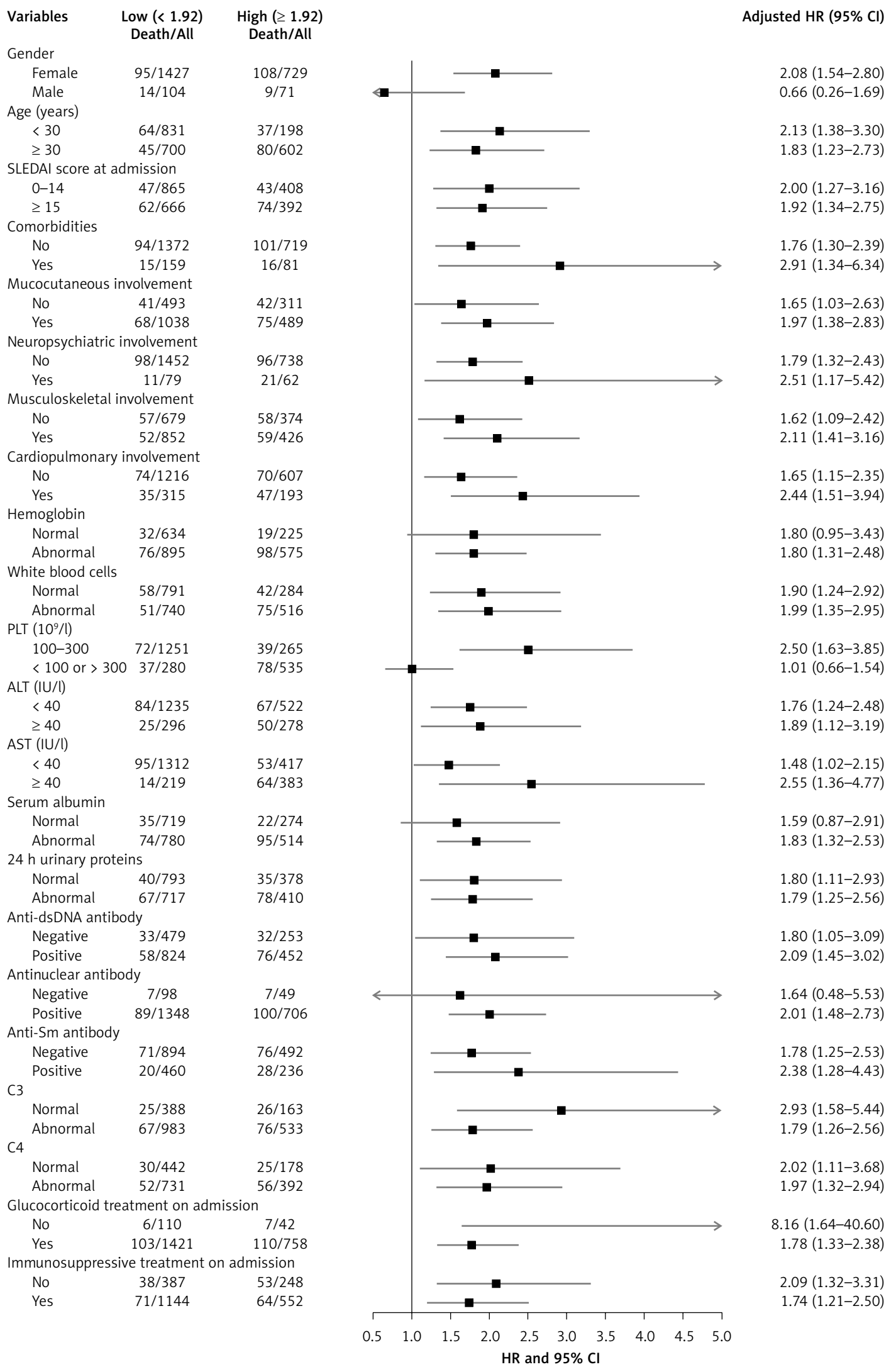

Figure 2. Hazard ratios for survival according to FIB-4 index stratified by clinical factors of systemic lupus erythematosus (SLE) patients. Models were adjusted for gender (male - 1, female - 0), SLEDAI score at admission (continuous), comorbidities (yes - 1, no - 0), glucocorticoid treatment on admission (yes - 1, no - 0) and immunosuppressive treatment on admission (yes -1, no -0 ), and the above adjusted variables would be excluded when it was the stratified variable 
A

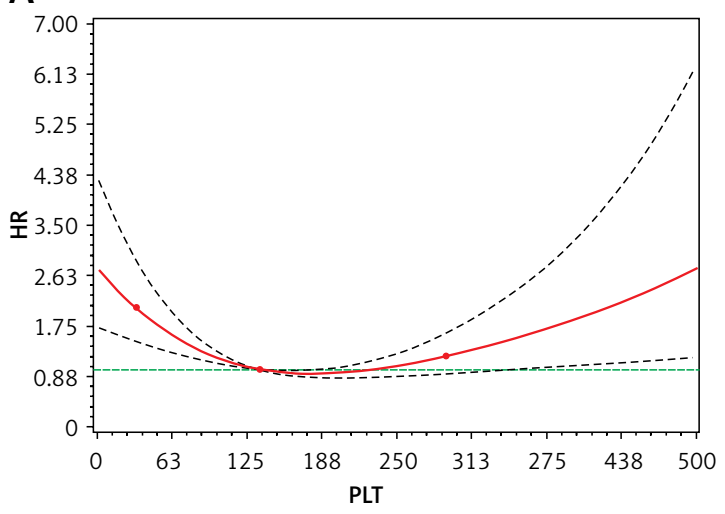

C

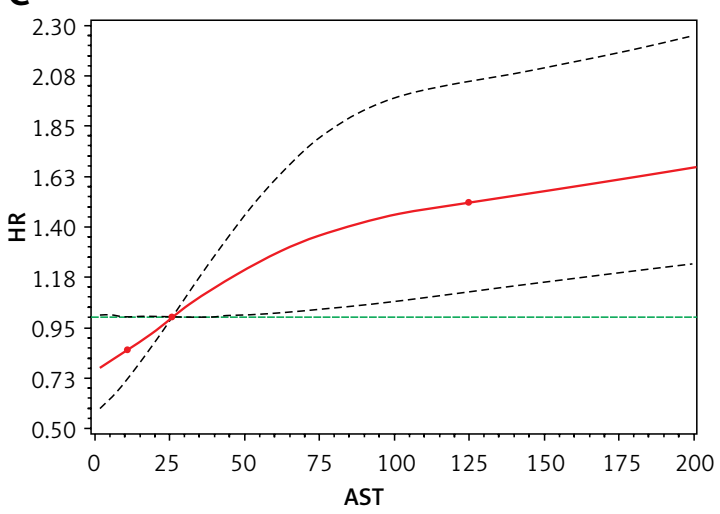

- Estimation
B

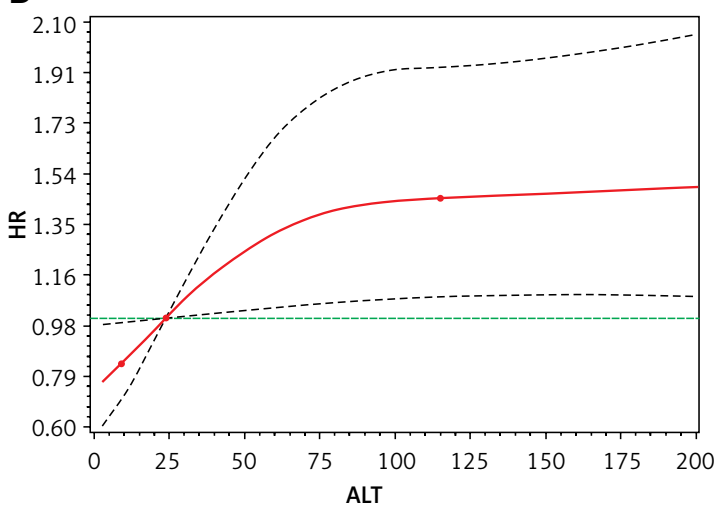

D

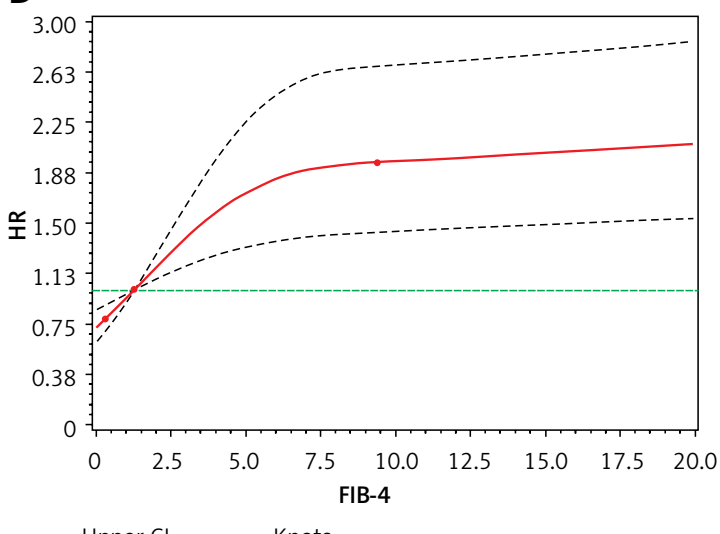

Figure 3. Dose-response association between FIB-4 index and mortality of systemic lupus erythematosus (SLE) patients. RCS plots were adjusted dose-response association between mortality of SLE patients and (A) PLT, (B) ALT, (C) AST, and (D) FIB-4 index. Each liver function index was coded using RCS function with three knots, located at the $5^{\text {th }}, 50^{\text {th }}$, and $95^{\text {th }}$ percentiles (represented by dots). Y-axis represents the adjusted HR for SLE death for individuals with any value compared to individuals with median. Dashed lines are $95 \% \mathrm{Cl}$. The $P$-values for overall association and nonlinear association were both $<0.001$ for PLT, 0.043 and 0.046 for ALT, $<0.001$ and 0.109 for AST, and both $<0.001$ for FIB-4 index

$\operatorname{dex}(\geq 1.29$ vs. $<1.29)$ was found associated with increased risks of adverse liver events including liver-related mortality $(\mathrm{HR}=4.24,95 \% \mathrm{Cl}: 2.19-$ 8.23) and cirrhosis development during a mean follow-up period of 15.47 years [20]. Moreover, two retrospective studies demonstrated that FIB-4 index predicted overall mortality and liver events, but both concern not very large cohorts with longterm follow-up to study disease-specific mortality $[30,31]$. One is a retrospective, international, multicenter cohort study, with a median of 8.7 years follow-up of 320 patients diagnosed with NAFLD, and the other is a retrospective Canadian cohort study with a median of 5 years of 148 patients who had liver biopsies and hepatic venous pressure gradient measurement. In this study with up to 15 years follow-up, we observed that 4 subjects died from liver diseases and all had a high FIB-4 index, so long-term observation would be needed in the future.

There is probably another reason why we cannot observe enough liver-related deaths, because competition risk exists in causes of death. Infections and organ involvement (mainly neuropsychiatric, renal and cardiopulmonary) affected by SLE are among the major causes of deaths among patients with SLE in Asian countries, which might produce the effects of competing risks on deaths of liver-related diseases [32]. Thus, in this study we observed that high FIB-4 index was mainly associated with the increased risk of specific mortality from infections, neuropsychiatric involvement, cardiopulmonary involvement and renal failure, though the latter two were not significant because of the limitation of small sample size.

The harm of liver-related diseases has been shown and affects other organs' function among SLE patients, which makes it possible to understand how increased FIB-4 index is associated with a poorer outcome of SLE. In this study, we found that high FIB-4 index was positively associated with other risk factors, such as SLEDAI score at admission, $24 \mathrm{~h}$ urinary proteins, neuropsychiatric and cardiopulmonary organ involvement 
Table III. Logistic regression analysis for high FIB-4 index

\begin{tabular}{|c|c|c|c|}
\hline Variables & OR & $95 \% \mathrm{Cl}$ & $P$-value \\
\hline Gender (female) & 1.34 & $0.98-1.83$ & 0.071 \\
\hline Age (years) & 1.06 & $1.06-1.07$ & $<0.001$ \\
\hline BMI $\left(\mathrm{kg} / \mathrm{m}^{2}\right)$ & 1.05 & $0.97-1.14$ & 0.213 \\
\hline SLEDAI score at admission & 1.03 & $1.02-1.04$ & $<0.001$ \\
\hline \multicolumn{4}{|l|}{ Laboratory tests: } \\
\hline Serum hemoglobin & 0.99 & $0.98-0.99$ & $<0.001$ \\
\hline White blood cells & 0.93 & $0.90-0.96$ & $<0.001$ \\
\hline Serum albumin $(g / l)$ & 0.99 & $0.98-1.00$ & 0.028 \\
\hline $24 \mathrm{~h}$ urinary proteins $(\mathrm{g})$ & 1.01 & $1.00-1.02$ & 0.037 \\
\hline \multicolumn{4}{|l|}{ Comorbidities: } \\
\hline All & 0.97 & $0.73-1.29$ & 0.845 \\
\hline Diabetes & 0.90 & $0.56-1.46$ & 0.668 \\
\hline Hypertension & 1.28 & $0.70-2.34$ & 0.419 \\
\hline Secondary Sjögren's syndrome & 1.00 & $0.51-1.96$ & 0.989 \\
\hline Pulmonary or upper respiratory infections & 0.63 & $0.30-1.36$ & 0.239 \\
\hline Others & 0.91 & $0.54-1.54$ & 0.729 \\
\hline \multicolumn{4}{|l|}{ Drugs used before admission: } \\
\hline Glucocorticoids & 0.32 & $0.04-2.65$ & 0.289 \\
\hline Immunosuppressive & 0.72 & $0.59-0.88$ & 0.001 \\
\hline \multicolumn{4}{|l|}{ Treatments on admission: } \\
\hline Glucocorticoids & 1.40 & $0.97-2.02$ & 0.074 \\
\hline Immunosuppressive & 0.75 & $0.62-0.91$ & 0.003 \\
\hline \multicolumn{4}{|l|}{ Organ involvement: } \\
\hline Mucocutaneous & 0.75 & $0.63-0.89$ & 0.001 \\
\hline Neuropsychiatric & 1.54 & $1.10-2.18$ & 0.013 \\
\hline Musculoskeletal & 0.91 & $0.77-1.08$ & 0.269 \\
\hline Cardiopulmonary & 1.23 & $1.00-1.51$ & 0.049 \\
\hline \multicolumn{4}{|l|}{ Positive autoantibodies: } \\
\hline Anti-dsDNA & 1.04 & $0.86-1.26$ & 0.698 \\
\hline Antinuclear antibody & 1.05 & $0.73-1.49$ & 0.799 \\
\hline Anti-Sm & 0.93 & $0.77-1.13$ & 0.473 \\
\hline \multicolumn{4}{|l|}{ Abnormal complement: } \\
\hline $\mathrm{C} 3$ & 1.29 & $1.05-1.59$ & 0.018 \\
\hline C4 & 1.33 & $1.08-1.65$ & 0.008 \\
\hline
\end{tabular}

BMI - body mass index, SLEDAI - SLE Disease Activity Index, FIB-4 - fibrosis-4. 
Z. Jin, X. Feng, D. Wang, W. Pan, L. Liu, M. Wu, H. Hu, X. Ding, H. Wei, Y. Zou, X. Qian, M. Wang, J. Wu, J. Tao, J. Tan, Z. Da, M. Zhang, J. Li, L. Sun

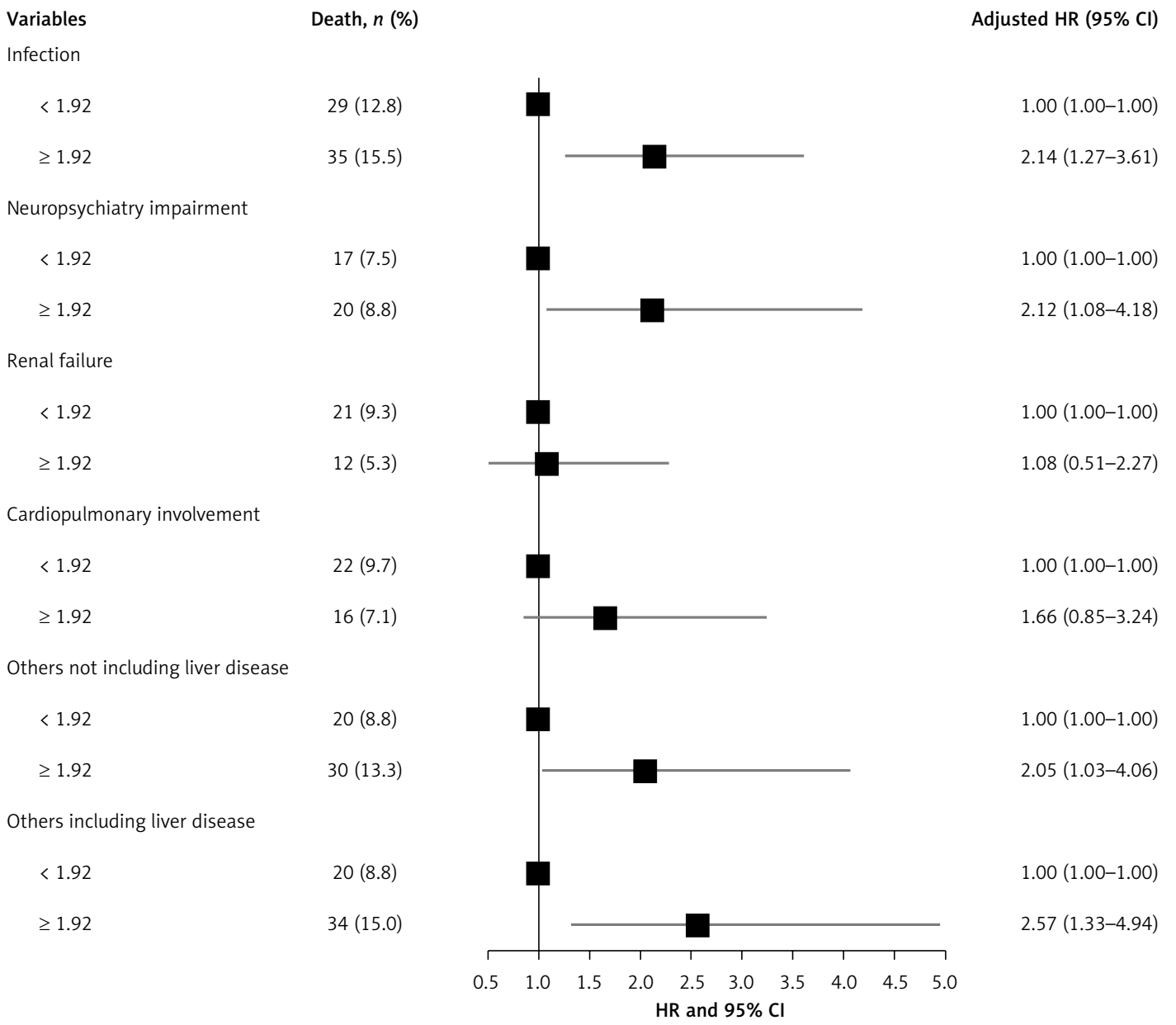

Figure 4. Association between FIB-4 index and cause-specific mortality among patients with systemic lupus erythematosus (SLE). Models were adjusted for gender (male - 1, female -0), SLEDAI score at admission (continuous), comorbidities (yes -1 , no - 0), glucocorticoid treatment on admission (yes - 1, no - 0) and immunosuppressive treatment on admission (yes - 1 , no - 0)

(Table III). In Western countries, cardiovascular involvements and malignancies have become the leading causes of death among Western patients with SLE $[11,12]$. In regard to cancers, a meta-analysis reported that liver cancer was more frequently observed in patients with SLE with a standardized incidence ratio (SIR) of $2.44(95 \%$ $\mathrm{Cl}: 1.46-4.05)$, compared to the general population [13]. Also the SIR of liver cancer was higher than the SIR observed for all the other cancers overall (1.16) or for lung (1.68), prostate (0.71) or bladder (1.78) cancer. As for cardiovascular involvement, a large body of evidence indicates that it is also associated with liver diseases, given that acute or chronic heart diseases can affect liver congestion and/or ischemia and impaired liver function can lead to heart failure [33, 34]. As for renal failure, high FIB-4 index has been suggested as an independent risk factor of prevalent chronic kidney disease in patients with NAFLD [15]. Even for neuropsychiatric involvement, accumulated epidemiological evidence has suggested that patients with elevated ALT level, liver cirrhosis, or liver disease have a significantly increased risk of intracranial hemorrhage and ischemic stroke [35, 36]. Also the mechanisms conferring the association are numerous, including endothelial dysfunction and vessel wall damage, inflammation, oxidative stress, and impaired hemostasis due to liver dysfunction [35]. Besides SLE itself, many studies have found that bacterial and fungal infections are common in patients with liver disease, especially among patients with liver cirrhosis and cirrhosis $[37,38]$. Several biological mechanisms have been proposed to explain the susceptibility to infection among patients with liver disease, including bacterial translocation from the intestine, impaired hepatic clearance mechanisms, and immune paralysis of circulating immune cells [39].

Several limitations of this study should be acknowledged. First, we used blood tests to assess the liver function among patients without a liver biopsy and imagological examination, as they are seldom performed on SLE patients for liver function examination in routine clinical practice. Thus, information on patients diagnosed with liv- 
Table IV. Joint effects of FIB-4 index and SLEDAI score at admission on hazard ratios for survival of patients with systemic lupus erythematosus (SLE)

\begin{tabular}{|c|c|c|c|c|}
\hline Variables & SLEDAI score & Death/All & OR $(95 \% \mathrm{Cl})$ & aOR $(95 \% \mathrm{Cl})^{\mathrm{a}}$ \\
\hline \multicolumn{5}{|l|}{ PLT $\left(10^{9} / l\right)$} \\
\hline $100-300$ & $0-14$ & $53 / 871$ & 1.00 & 1.00 \\
\hline$<100$ or $>300$ & $0-14$ & $37 / 402$ & $1.51(0.96-2.39)$ & $1.54(0.97-2.43)$ \\
\hline $100-300$ & $\geq 15$ & $58 / 645$ & $1.71(1.14-2.54)$ & $1.88(1.25-2.81)$ \\
\hline \multirow[t]{2}{*}{$<100$ or $>300$} & $\geq 15$ & $78 / 413$ & $3.75(2.58-5.45)$ & $3.83(2.63-5.58)$ \\
\hline & & & \multicolumn{2}{|c|}{ Interaction: $p=0.339$} \\
\hline \multicolumn{5}{|l|}{ ALT (IU/I) } \\
\hline$<40$ & $0-14$ & $60 / 967$ & 1.00 & 1.00 \\
\hline$\geq 40$ & $0-14$ & $30 / 306$ & $1.59(0.98-2.56)$ & $1.60(0.99-2.59)$ \\
\hline$<40$ & $\geq 15$ & $91 / 790$ & $2.18(1.54-3.09)$ & $2.34(1.65-3.34)$ \\
\hline \multirow[t]{2}{*}{$\geq 40$} & $\geq 15$ & $45 / 268$ & $3.32(2.18-5.04)$ & $3.52(2.31-5.36)$ \\
\hline & & & \multicolumn{2}{|c|}{ Interaction: $p=0.846$} \\
\hline \multicolumn{5}{|l|}{ AST (IU/I) } \\
\hline$<40$ & $0-14$ & $65 / 981$ & 1.00 & 1.00 \\
\hline$\geq 40$ & $0-14$ & $25 / 292$ & $1.48(0.90-2.44)$ & $1.51(0.92-2.49)$ \\
\hline$<40$ & $\geq 15$ & $83 / 748$ & $2.01(1.42-2.86)$ & $2.18(1.53-3.10)$ \\
\hline \multirow[t]{2}{*}{$\geq 40$} & $\geq 15$ & $53 / 310$ & $3.36(2.27-4.98)$ & $3.59(2.41-5.33)$ \\
\hline & & & \multicolumn{2}{|c|}{ Interaction: $p=0.785$} \\
\hline \multicolumn{5}{|l|}{ FIB-4 index } \\
\hline$<1.92$ & $0-14$ & $47 / 865$ & 1.00 & 1.00 \\
\hline$\geq 1.92$ & $0-14$ & $43 / 408$ & $1.95(1.24-3.07)$ & $1.96(1.25-3.09)$ \\
\hline$<1.92$ & $\geq 15$ & $62 / 666$ & $2.01(1.35-3.00)$ & $2.16(1.44-3.23)$ \\
\hline \multirow[t]{2}{*}{$\geq 1.92$} & $\geq 15$ & $74 / 392$ & $4.21(2.84-6.23)$ & $4.24(2.86-6.31)$ \\
\hline & & & \multicolumn{2}{|c|}{ Interaction: $p=0.996$} \\
\hline
\end{tabular}

${ }^{a}$ Adjusted for sex (male - 1, female - 0), age (continuous, except for variables of FIB-4 index), comorbidities (yes - 1, no - 0), glucocorticoid treatment on admission (yes - 1, no - 0), immunosuppressive treatment on admission (yes - 1, no - 0). PLT - platelet, ALT - alanine aminotransferase, AST - aspartate aminotransferase, FIB-4 - fibrosis-4.

er cirrhosis was lacking. However, previous studies have validated the performance characteristics of scoring system methods in predicting hepatic fibrosis and overall mortality, regardless of etiology [17, 18, 27, 40-46]. Moreover, compared to the Child-Pugh and Model for End-Stage Liver Disease (MELD) scores, the FIB-4 index was better in predicating hepatic decompensation and HCC in HCV-positive persons, and showed no differences in predictive ability for mortality $[47,48]$. Further studies still should be conducted to validate the performance of the FIB-4 index in predicating mortality among SLE patients. Second, we observed a very low percentage (less than 1\%) of viral infections and alcohol drinking, because the majority of (92.5\%) of SLE patients were fe- male in this study, and the prevalence of alcohol drinking (9.4\%) and viral infections of HBV (5.7\%) and HCV (0.4\%) was low among Chinese females in general [49]. Consistently, a study on 206 SLE patients reported that the causes of liver dysfunction were limitedly due to viral infections (0.8\%), alcohol (1.6\%), and fatty liver (17.9\%), but mainly due to either SLE itself (30.9\%) or drugs (28.5\%) [4]. Moreover, we cannot include all risk factors of liver diseases due to the nature of the retrospective cohort study design. Thus, the causes of liver enzyme abnormalities were overall in this study. In spite of those limitations, however, the present study has several strengths as follows: 1 ) it was a large-scale multicenter cohort study; 2) having 15 years of mortality follow-up; 3 ) the key vari- 
A

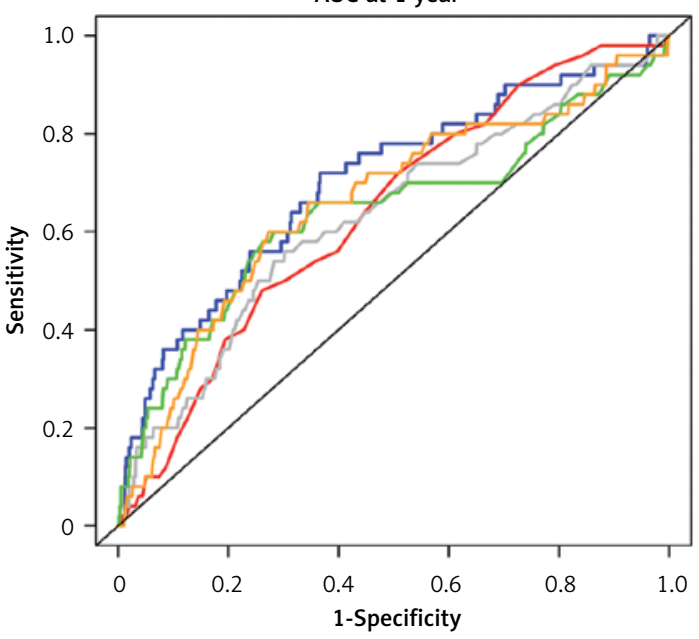

C

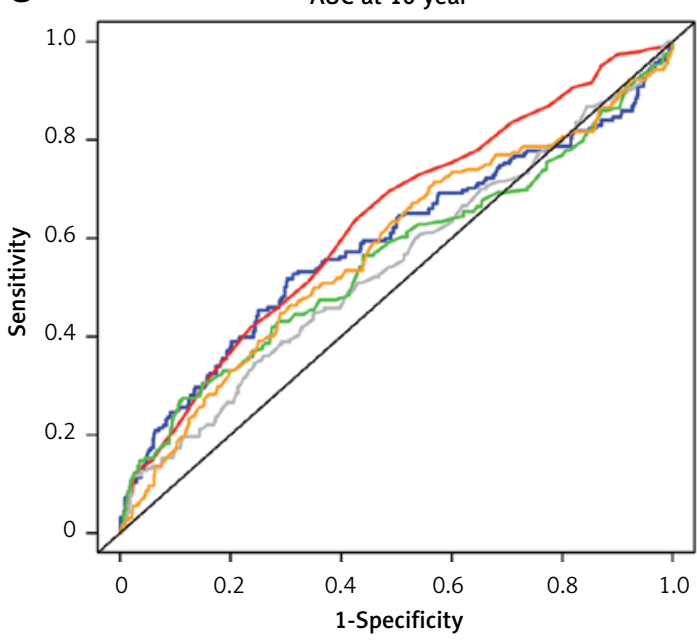

- SLEDAI score: 0.63 (95\% Cl: 0.58-0.68)

- FIB-4 index: $0.59(95 \% \mathrm{Cl}: 0.53-0.65)$

- ALT: 0.55 (95\% Cl: 0.49-0.60)

- AST: $0.56(95 \% \mathrm{Cl}: 0.50-0.62)$

- PLT: 0.58 (95\% Cl: 0.52-0.63)

B

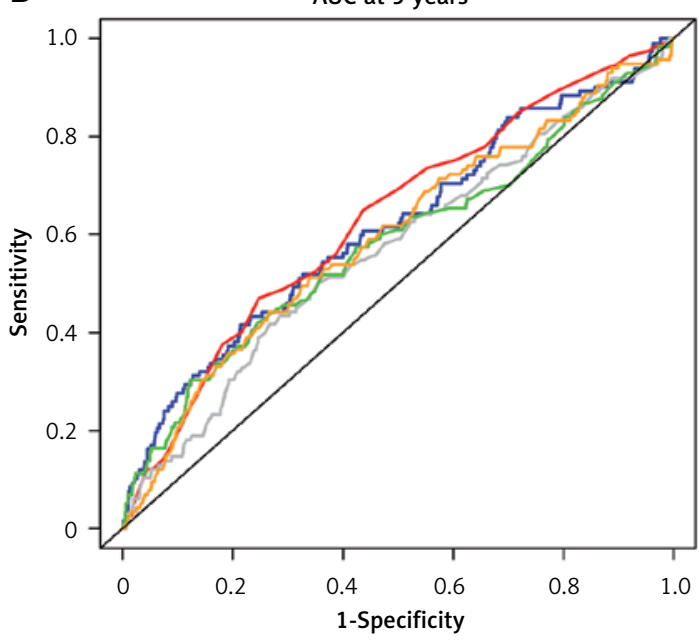

- SLEDAI score: 0.63 (95\% Cl: 0.58-0.69)

- FIB-4 index: 0.62 (95\% Cl: 0.56-0.67)

- ALT: 0.57 (95\% Cl: 0.51-0.63)

- AST: 0.58 (95\% Cl: 0.52-0.64)

- PLT: 0.60 (95\% Cl: 0.54-0.65)

D

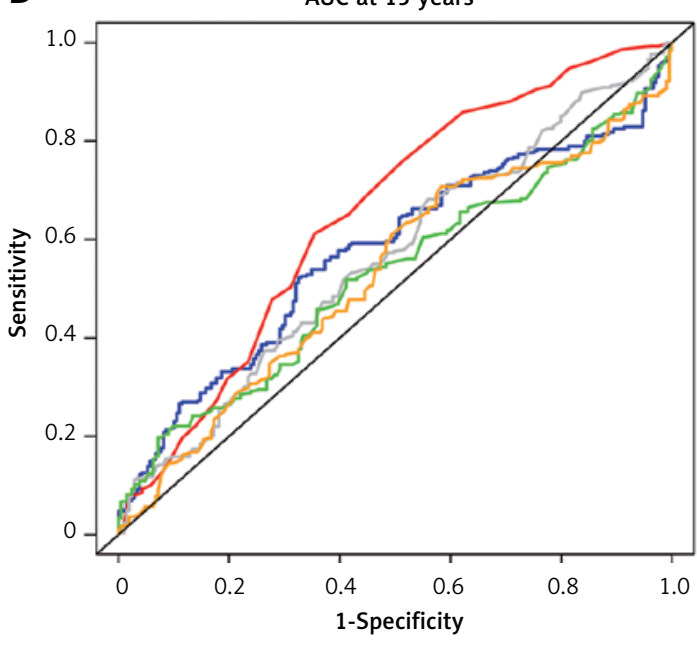

- SLEDAI score: 0.65 (95\% Cl: 0.59-0.71)

— FIB-4 index: 0.57 (95\% Cl: 0.51-0.64)

- ALT: 0.56 (95\% Cl: 0.50-0.62)

- AST: 0.53 (95\% Cl: 0.46-0.59)

- PLT: 0.53 (95\% Cl: 0.46-0.60)

Figure 5. Estimating and comparing time-dependent AUCs between FIB-4 index and SLEDAI score at (A) 1, (B) 5 ,

(C) 10 and (D) 15 years from our systemic lupus erythematosus (SLE) cohort study

ables were from laboratory tests and detailed information on various mortality risk factors of SLE was collected; 4) both multivariate and stratified analyses were carried out for excluding potential confounding factors.

In conclusion, this retrospective cohort study is the first to report an association between higher FIB4 index and increased overall mortality of SLE with a nonlinear dose-response relationship, and found no significant differences of AUCs between FIB-4 index and SLEDAI score. The FIB-4 index may poten- tially serve as an independent predictor for prognosis in SLE, and warrants further in-depth investigations.

\section{Funding}

This work was supported by the Major International (Regional) Joint Research Project (No. 81720108020), the Jiangsu Province Major Research and Development Program (No. BE2015602), Jiangsu Province 333 Talent Grant (No. BRA2016001), the National Key Research and Development Program of China (No. 2017YFC0909003), and the 
Key Program of National Natural Science Foundation of China (No. 81930043).

\section{Acknowledgments}

The authors would like to thank all investigators and site staff of local hospitals who participated in this study and Cinkate Corp for their assistance in helping build and manage our database website.

\section{Conflict of interest}

The authors declare no conflict of interest.

\section{References}

1. Cervera R, Khamashta MA, Font J, et al. Systemic lupus erythematosus: clinical and immunologic patterns of disease expression in a cohort of 1,000 patients. The European Working Party on Systemic Lupus Erythematosus. Medicine (Baltimore) 1993; 72: 113-24.

2. Van HB. The spectrum of liver disease in systemic lupus erythematosus. Neth J Med 1996; 48: 244-53.

3. Runyon BA, LaBrecque DR, Anuras $S$. The spectrum of liver disease in systemic lupus erythematosus. Report of 33 histologically-proved cases and review of the literature. Am J Med 1980; 69: 187-94.

4. Takahashi A, Abe K, Saito R, et al. Liver dysfunction in patients with systemic lupus erythematosus. Intern Med 2013; 52: 1461-5.

5. Brewer BN, Kamen DL. Gastrointestinal and hepatic disease in systemic lupus erythematosus. Rheum Dis Clin N Am 2018; 44: 165-75.

6. Leggett BA. The liver in systemic lupus erythematosus. J Gastroenterol Hepatol 1993; 8: 84-8.

7. Mak A, Cheung WL, Chiew HJ, Liu Y, Chun-man Ho R. Global trend of survival and damage of systemic lupus erythematosus: meta-analysis and meta-regression of observational studies from the 1950s to 2000s. Semin Arthritis Rheu 2012; 41: 830-9.

8. Wang Z, Wang Y, Zhu R, et al. Long-term survival and death causes of systemic lupus erythematosus in China: a systemic review of observational studies. Medicine (Baltimore) 2015; 94: e794.

9. Bataller R, Sancho-Bru P, Gines P, Brenner DA. Liver fibrogenesis: a new role for the renin-angiotensin system. Antioxid Redox Sign 2005; 7: 1346-55.

10. Bataller R, Brenner DA. Liver fibrosis. J Clin Invest 2005; 115: 209-18.

11. Rees F, Doherty M, Grainge MJ, et al. Mortality in systemic lupus erythematosus in the United Kingdom 1999-2012. Rheumatology (Oxford) 2016; 55: 854-60.

12. Thomas G, Mancini J, Jourde-Chiche N, et al. Mortality associated with systemic lupus erythematosus in France assessed by multiple-cause-of-death analysis. Arthritis Rheumatol 2014; 66: 2503-11.

13. Ni J, Qiu LJ, Hu LF, et al. Lung, liver, prostate, bladder malignancies risk in systemic lupus erythematosus: evidence from a meta-analysis. Lupus 2014; 23: 284-92.

14. Jablonska J, Cielecka-Kuszyk J, Mikula T, Kozłowska J, Wiercińska-Drapało A. Hepatopathy of unknown etiology - is liver biopsy a good tool in differential diagnosis? Arch Med Sci 2019; 15: 1462-7.

15. Xu HW, Hsu YC, Chang CH, Wei KL, Lin CL. High FIB-4 index as an independent risk factor of prevalent chronic kidney disease in patients with nonalcoholic fatty liver disease. Hepatol Int 2016; 10: 340-6.

16. Shah AG, Lydecker A, Murray K, Tetri BN, Contos MJ, Sanyal AJ; Nash Clinical Research Network. Comparison of noninvasive markers of fibrosis in patients with nonalcoholic fatty liver disease. Clin Gastroenterol Hepatol 2009; 7: 1104-12.

17. Salomone F, Micek A. Simple scores of fibrosis and mortality in patients with NAFLD: a systematic review with meta-analysis. J Clin Med 2018; 7: E219.

18. Unalp-Arida A, Ruhl CE. Liver fibrosis scores predict liver disease mortality in the United States population. Hepatology 2017; 66: 84-95.

19. Kim D, Kim WR, Kim HJ, Therneau TM. Association between noninvasive fibrosis markers and mortality among adults with nonalcoholic fatty liver disease in the United States. Hepatology 2013; 57: 1357-65.

20. Tseng TC, Liu CJ. Fibrosis-4 index predicts cirrhosis risk and liver-related mortality in 2075 patients with chronic HBV infection. Aliment Pharmacol Ther 2018; 47: 1480-9.

21. Gladman DD, Ibanez D, Urowitz MB. Systemic lupus erythematosus disease activity index 2000. J Rheumatol 2002; 29: 288-91.

22. Feng X, Zou Y, Pan W, et al. Prognostic indicators of hospitalized patients with systemic lupus erythematosus: a large retrospective multicenter study in China. J Rheumatol 2011; 38: 1289-95.

23. Feng X, Pan W, Liu L, et al. Prognosis for hospitalized patients with systemic lupus erythematosus in China: 5-year update of the Jiangsu cohort. PLoS One 2016; 11: e0168619.

24. Hochberg MC. Updating the American College of Rheumatology revised criteria for the classification of systemic lupus erythematosus. Arthritis Rheum 1997; 40: 1725.

25. Tan EM, Cohen AS, Fries JF, et al. The 1982 revised criteria for the classification of systemic lupus erythematosus. Arthritis Rheum 1982; 25: 1271-7.

26. China National Health and Family Planning Commission. Act on thical review system of biomedical research involving human subjects. Available at: http://www.gov. cn/gongbao/content/2017/content_5227817.htm.

27. Sterling RK, Lissen E, Clumeck N, et al. Development of a simple noninvasive index to predict significant fibrosis in patients with HIV/HCV coinfection. Hepatology 2006; 43: 1317-25.

28. Gladman D, Ginzler E, Goldsmith C, et al. The development and initial validation of the Systemic Lupus International Collaborating Clinics/American College of Rheumatology damage index for systemic lupus erythematosus. Arthritis Rheum 1996; 39: 363-9.

29. Blanche P, Dartigues JF, Jacqmin-Gadda H. Estimating and comparing time-dependent areas under receiver operating characteristic curves for censored event times with competing risks. Stat Med 2013; 32: 5381-97.

30. Angulo P, Bugianesi E, Bjornsson ES, et al. Simple noninvasive systems predict long-term outcomes of patients with nonalcoholic fatty liver disease. Gastroenterology 2013; 145: 782-9.e4.

31. Sebastiani G, Alshaalan R, Wong P, et al. Prognostic value of non-invasive fibrosis and steatosis tools, hepatic venous pressure gradient (HVPG) and histology in nonalcoholic steatohepatitis. PLoS One 2015; 10: e0128774.

32. Jakes RW, Bae SC, Louthrenoo W, Mok CC, Navarra SV, Kwon N. Systematic review of the epidemiology of systemic lupus erythematosus in the Asia-Pacific region: prevalence, incidence, clinical features, and mortality. Arthritis Care Res (Hoboken) 2012; 64: 159-68. 
33. Møller S, Dumcke CW, Krag A. The heart and the liver. Expert Rev Gastroenterol Hepatol 2009; 3: 51-64.

34. Sao R, Aronow WS. Association of non-alcoholic fatty liver disease with cardiovascular disease and subclinical atherosclerosis. Arch Med Sci 2018; 14: 1233-44.

35. Kim HC, Oh SM, Pan WH, et al. Association between alanine aminotransferase and intracerebral hemorrhage in East Asian populations. Neuroepidemiology 2013; 41: 131-8.

36. Parikh NS, Merkler AE, Schneider Y, Navi BB, Kamel H. Discharge disposition after stroke in patients with liver disease. Stroke 2017; 48: 476-8.

37. Wang C, Ma DQ, Luo S, et al. Incidence of infectious complications is associated with a high mortality in patients with hepatitis B virus-related acute-on-chronic liver failure. World J Clin Cases 2019; 7: 2204-16.

38. Ekpanyapong S, Reddy KR. Infections in cirrhosis. Curr Treat Options Gastroenterol 2019; 17: 254-70.

39. Selvapatt N, Singanayagam A, Wendon J, Antoniades CG. Understanding infection susceptibility in patients with acute-on-chronic liver failure. Intensive Care Med 2014; 40: 1363-6.

40. Vallet-Pichard A, Mallet V, Pol S. FIB-4: a simple, inexpensive and accurate marker of fibrosis in HCV-infected patients. Hepatology 2006; 44: 769; author reply 769-70.

41. Vallet-Pichard A, Mallet V, Nalpas B, et al. FIB-4: an inexpensive and accurate marker of fibrosis in $\mathrm{HCV}$ infection. comparison with liver biopsy and fibrotest. Hepatology 2007; 46: 32-6.

42. Kim BK, Kim DY, Park JY, et al. Validation of FIB-4 and comparison with other simple noninvasive indices for predicting liver fibrosis and cirrhosis in hepatitis B virus-infected patients. Liver Int 2010; 30: 546-53.

43. Takyar V, Surana P, Kleiner DE, et al. Noninvasive markers for staging fibrosis in chronic delta hepatitis. Aliment Pharmacol Ther 2017; 45: 127-38.

44. Sumida $\mathrm{Y}$, Yoneda M, Hyogo $\mathrm{H}$, et al. Validation of the FIB4 index in a Japanese nonalcoholic fatty liver disease population. BMC Gastroenterol 2012; 12: 2.

45. Chrostek L, Panasiuk A. Liver fibrosis markers in alcoholic liver disease. World J Gastroenterol 2014; 20: 8018-23.

46. Kawaguchi T, Inokuchi T, Honma T, et al. Factors associated with advanced hepatic fibrosis in patients with various internal diseases: A multicenter communitybased survey. Hepatol Res 2018; 48: 882-92.

47. Butt AA, Ren Y, Lo Re V, 3rd, Taddei TH, Kaplan DE. Comparing Child-Pugh, MELD, and FIB-4 to predict clinical outcomes in hepatitis $C$ virus-infected persons: results from ERCHIVES. Clin Infect Dis 2017; 65: 64-72.

48. Peng Y, Qi X, Guo X. Child-Pugh Versus MELD score for the assessment of prognosis in liver cirrhosis: a systematic review and meta-analysis of observational studies. Medicine (Baltimore) 2016; 95: e2877.

49. Jin ZY, Liu X, Ding YY, Zhang ZF, He N. Cancer risk factors among people living with HIV/AIDS in China: a systematic review and meta-analysis. Sci Rep 2017; 7: 4890. 